\title{
RECOMMENDATIONS FOR ADDRESSING PRIORITY IO SCIENCE IN THE NEXT DECADE
}

Missions, Technology, Instruments, Earth-based Observations, Research and Analysis, Workforce, and More

A WHITE PAPER TO THE 2023-2032 PLANETARY SCIENCE AND ASTROBIOLOGY DECADAL SURVEY

\section{James Tuttle Keane}

California Institute of Technology, Jet Propulsion Laboratory james.t.keane@jpl.nasa.gov

Alexandra A. Ahern (Stony Brook University) · Fran Bagenal (L (Pennsylvania State University) · Patricio Becerra (Universität Bern) · Tanguy Bertrand (NASAAmes Research Center) · Ross A. Beyer (SETI Institute, NASA Ames Research Center) - Carver J. Bierson (University of California Santa Cruz) - Michael T. Bland (United States Geological Survey) - Doris Breuer (Deutsches Zentrum für Luft- und Raumfahrt e.V.) · Ashley G. Davies (California Institute of Technology, Jet Propulsion Laboratory) · Katherine de Kleer (California Institute of Technology) - Imke de Pater (University of California Berkeley) - Daniella N. DellaGiustina (University of Arizona) - Tilmann Denk . (Deutsches Zentrum für Luft- und Raumfahrt e.V.) · Ariana Echevarria (University of Illinois at Urbana-Champaign) · Catherine M. Elder (California Institute of Technology, Jet Propulsion Laboratory) - Lori M. Feaga (University of Maryland, College Park) - Cesare Grava (Southwest Research Institute) Patricia M. Gregg (University of Illinois at Urbana-Champaign). Tracy K.P. Gregg (University of Buffalo) - Christopher W. Hamilton

Camilla D.K. Harris (University of Michigan) - Walter M. Harris (University of Arzona) · Hamish, C.F.C. Hay Laboratory) - Amanda R. Hendrix (Planetary Science Institute) - Sarah M. Hörst (Johns Hopkins University) • Rowan Huang (Brigham Young University) Andréa C.G. Hughes (Embry-Riddle Aeronautical Univerșity) · Kandis Lea Jessup (Southwest Research Institute, Regis University) · Xianzhe Jia of Michigan) · Lauren M. Jozwiak (Johns Hopkins University Applied Physics Laboratory) - Laura Kerber (California Instititute of Technology, Jet Pr - Laszlo P. Kestay (United States Geological Survey) - Krishan K. Khurana (Uhin

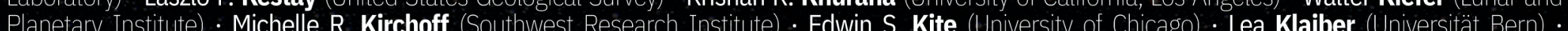

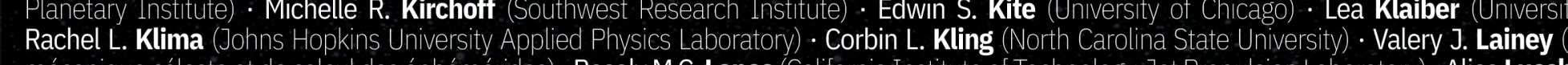
- mécanique céleste et de calcul des éphémérides) - Rosaly M.C. Lopes (California Institute of Technology, Jet Propulsion Laboratory) - Alice Lucchetti (INAF : Astronomical Observatory of Padova) · Kathleen E. Mandt (Johns. Hopkins University Applied. Physics Laboratory) - Isamu Matsuyama (

Arizona) - Christine McCarthy (Columbia University) · Alfred S. McEwen (University of Arizona) - Melissa A. McGrath (SETI Institute) - Laurent G.J. Montési (University of Maryland, College Park) - Julieanne I. Moses (Space Science Institute) - Arielle Moullet (National Radio Astronomy Observatory) · Quentin Nénon (University of California, Berkeley) · Gregory A. Neumann (NASA Goddard Space Flight Center) · Marc F. Neveu (University of Maryland, College Park, NASA Goddard Space Flight Center) · Francis Nimmo (University of California, Santa Cruz) · John W. Noonan (University of Arizona) - Maurizio Pajola (INAF Astronomical Observatory of Padova) · Mark P. Panning (California Institute of Technology, Jet Propulsion Laboratory) · Ryan S. Park (California Institute of Technology, Jet Propulsion Laboratory) - Anne Pommier (University of California, San Diego) - Lynnae C. Quick (NASA Goddard Space Flight Center) * Jani Radebaugh (Brigham Young University) · Julie A. Rathbun (Planetary Science Institute) · Kurt D. Retherford (Southwest Résearch Institute) :
James H. Roberts (Johns Hopkins University Applied Physics Laboratory) - Elias Roussos (Max Planck Institute for Solar System Research) P Paul M. Schenk (Lunar and Planetary. Institute) · Nick M. Schneider (University of Colorado, Boulder) · Joe W. Schools (University of Maryland, College Park) · Rohan Sood (University of Alabama) : John R. Spencer (Southwest Research Institute) - Dan C. Spencer (University of Oxford) - Gregor Steinbrügge (Stanford University) - Ali H. Sulaiman (University of Iowa) - Sarah S. Sutton (University of Arzona) - Antony Trinh (University of Arizona) - Constantine C.C. Tsang (Southwest Research Institute) · Janet Vertesi (Princeton University) • Audrey Vorburger (University of Bern) · Joseph H. Westlake (Johns Hopkins University Applied Physics Laboratory) · David A. Williams

A portion of this research was carried out at the Jet Propulsion Laboratory, California Institute of Technology, under a contract with the National Aeronautics and Space Administration (80NM0018D0004). (52020. All rights reserved. The information presented in this white paper is pre-decisional and is provided for planning and discussion purposes only. 
SUMMARY: Io is a priority destination for solar system exploration, as it is the best natural laboratory to study the intertwined processes of tidal heating, extreme volcanism, and atmospheremagnetosphere interactions. lo exploration is relevant to understanding terrestrial planets and moons (including the early Earth), ocean worlds, and exoplanets across the cosmos. The scope and importance of science questions at lo necessitates a broad portfolio of research and analysis, telescopic observations, and planetary missions-including a dedicated New Frontiers class lo mission.

Recommendation 1: We strongly recommend a dedicated New Frontiers class lo mission for the next decade. As outlined in a companion white paper, The Science Case for $I o$, Io is a priority destination for future exploration. There is much that can only be learned through detailed in situ measurements and observations from a dedicated Io mission. Even as nextgeneration telescopes come online, we are unlikely to achieve spatial resolutions better than tens of kilometers, and we cannot make observations of Io's poles or night hemisphere. There are also entire suites of in situ measurements which are simply not possible without visiting Io-like measuring Io's gravity and magnetic fields, or sampling its plumes and atmosphere. Furthermore, a dedicated mission to Io in the coming decade is timely, as it would enhance the science return from current and future Jupiter-system missions (Juno, Europa Clipper, JUICE), enabling true system science and contemporaneous investigations of the tidal and orbital evolution of the entire Jovian system. A mission to Io could also inform and guide forthcoming exoplanet observations with next-generation telescopes, and inform our understanding of the origins of life through implications for the early Earth and tidally heated ocean worlds like Europa, Enceladus, and Titan.

Box 1 outlines a notional mission concept for a dedicated Io mission that could plausibly address all of the Priority Science Questions outlined in The Science Case for Io. This "Io Observer" concept is an amalgam of multiple different concepts, ideas, and mission proposals ${ }^{1-16}$. While we highlight one particular concept we encourage the decadal survey to allow flexibility in implementation. The past decade is characterized by mission selections that exemplify how new, innovative, and bold ideas have the power to transform the field (e.g., Dragonfly ${ }^{17}$ ). A Jupiterorbiting, multiple-flyby, Io Observer may be the prevailing approach at this time, but out-of-thebox concepts may be capable of addressing a preponderance of Priority Science Questions, including orbiters, landers, impactors, and distant observers.

The cost of the notional Io Observer mission concept outlined in Box 1 requires a detailed study by the decadal survey (see Recommendation 2). Io mission concepts have previously targeted either the Discovery program ${ }^{1,3,7,10,12,15}$ or the New Frontiers program ${ }^{2,6,8-9,13-14}$, with sporadic discussion of Flagship class Io missions ${ }^{4-5}$. Io exploration is possible in all cost regimes; the question is: what is the best trade between science and cost? Discovery class Io missions tend to focus on addressing a subset of possible Io Priority Science Questions (consistent with the scope of the Discovery program, which generally address targeted science investigations). New Frontiers class Io missions have potential to address all of the Priority Science Questions, and fully realize the cross-cutting planetary science potential of Io. Flagship class Io missions could completely address all Priority Science Questions, but may be unrealistic given other priorities in planetary science. Based on the scope of the Io science and the implementation challenges, we recommend the decadal survey consider Io as a priority for the New Frontiers program-consistent with past decadal surveys, midterm reports, reports from the Committee on Astrobiology and Planetary Science ${ }^{18-20}$. Put simply, Io offers New Frontiers caliber science.

At the time of writing this report, NASA is evaluating a proposed Discovery class mission to Io: Io Volcano Observer $(I V O)^{15}$. Owing to the ongoing competition, we refrain from discussing 
Box 1: Notional New Frontiers class "lo Observer" mission concept.

Science: Priority Science Questions for lo exploration are detailed in the companion white paper, The Science Case for 10 Exploration (Table 1).

Orbit: Jupiter-centric, eccentric orbit, with a perijove near lo-enabling multiple close low altitude ( $100 \mathrm{~km})$ flybys of lo. The orbital period (i.e., flyby cadence) and total mission duration (i.e., number of flybys) requires a trade study quantifying radiation dosage, fuel, cost, and science capabilities. Most past studies assume a 1-month orbital period and 10-50 flybys. With modern radiation mitigation techniques (see Recommendation 2), and a suitable choice of orbit, it is possible to build a multiple-flyby mission that receives less total radiation than Europa Clipper10.

Power: Solar power. There have been substantial improvements in solar power technologies for missions to the outer solar system and in high-radiation environments (see Recommendation 2).

Payload: There are a vast number of science instruments relevant to lo exploration, and the exact number and choice of instruments requires a detailed trade study. Here we list a set of high-heritage instruments that could address the Priority Science Questions outlined in The Science Case for lo Exploration. An lo Observer could plausibly have 3-5 instruments. Instruments are not ranked.

- Narrow-angle, multi-color, visible imaging camera (heritage: Europa Clipper EIS) for observing geologic features at small scales $(<1 \mathrm{~km})$, acquiring geodetic images, stereo for local topography, and high-cadence image sequences (i.e., movies) for monitoring active phenomena like plumes and eruptions.

- Wide-angle, multi-color, visible imaging camera (heritage: Europa Clipper EIS, New Horizons MVIC) for mapping at regional and global scales, providing geologic context for high-resolution datasets, change detection, and global topography.

- Thermal infrared radiometer (heritage: LRO Diviner, BepiColombo MERTIS) for measuring the thermal emission of lo's lava flows and plains, measuring lo's heat flow at both global and local scales, constraining $\mathrm{SO}_{2}$ frost temperatures, and regolith properties.

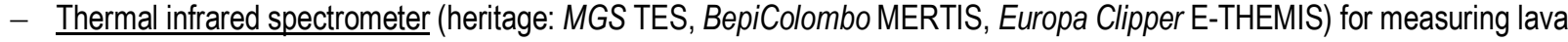
temperatures, characterizing the silicate mineralogy of the crust and lava flows, and characterizing lo's atmosphere.

- Near-infrared spectrometer (heritage: MRO CRISM, Europa Clipper MISE) for measuring mafic mineralogy of lo's surface, major cation composition $(\mathrm{Mg} / \mathrm{Fe}$ ratio), oxidation state, and the composition of sulfur species, and probing atmosphere composition and trace species.

- Ultraviolet spectrometer (heritage: Galileo UVS, Cassini UVIS, New Horizons Alice, MAVEN IUVS) for characterizing lo's atmosphere, plumes, auroral emissions, and plasma torus.

- Magnetometer (heritage: Galileo MAG, Cassini MAG, JUICE J-MAG, Europa Clipper Magnetometer) for measuring the intrinsic and induced magnetic field of lo and testing hypotheses about lo's internal structure (e.g., the presence of a magma ocean).

- Plasma and radiation suite (heritage: Galileo EPD/HIC/PLS/PWS, Cassini CAPS/MIMI/RPWS, JUICE PEP/RPWI/RADEM, Europa Clipper PIMS) for measuring the temporal and spatial distribution, speciation, and properties of charged particles, radio and plasma waves, and dust in the Jupiter system. These measurements are critical to interpreting magnetic field experiments, and for deciphering the complex interactions between lo and the Jovian magnetosphere and plasma environment.

- Laser altimeter (heritage: MGS MOLA, LRO LOLA, MESSENGER MLA, OSIRIS-REX OLA, JUICE GALA) for precisely measuring topography, small-scale mass movements, tidal flexure, libration, obliquity, and other geodetic quantities.

- Mass spectrometer (heritage: Galileo probe, Cassini INMS, Europa Clipper MASPEX, Rosetta ROSINA, JUICE NMS) for determining the composition and isotopic abundances of lo's atmosphere and plumes, and Jupiter's magnetosphere.

- Radio and gravity science (heritage: most planetary missions) for determining the gravity field of lo, astrometry, and atmospheric science.

In addition to these high-heritage instruments, there are a number of next-generation instruments that show significant promise for lo exploration (see Recommendation 3). However, while these next-generation instruments are exciting, we note that no new technologies are required for a modern lo mission that addresses a preponderance of the Priority Science Questions about lo.

IVO in detail, other than to note that it has the potential to address a preponderance of the Priority Science Questions outline in The Science Case for Io Exploration. Although IVO has been selected for Phase-A study, it has not yet been selected for flight. If $I V O$ is selected for flight, then the decadal survey would need to reevaluate relevance for New Frontiers. 
Recommendation 2: We recommend that the decadal survey perform a new, fullycosted, New Frontiers class lo mission study, reflecting recent scientific and technological advances described in this report. Despite the range of previous concepts for Io exploration ${ }^{1-16}$, there is legitimate need for a new New Frontiers class Io mission concept study. Both the decadal midterm review and pre-decadal reports ${ }^{19-20}$ advocated for new studies in light of recent scientific and technological advances. The only publicly available and fully-costed Io mission concept study is the 2010 Io Observer study performed for the previous decadal survey ${ }^{6}$. This study utilized a Jupiter-orbiting, multiple flyby architecture, carried 3-5 instruments, and cost between $\$ 958 \mathrm{M}-\$ 1.1 \mathrm{~B}$. While the 2010 Io Observer study was compelling at the time, it is now outdated due to a combination of scientific and technological advances. All other mission concepts from the past decade have either been preliminary trade studies ${ }^{8,9,11,13-14}$, or competed mission proposals where key details and trade studies are not public (e.g., $I V O^{7,10,12,15}$ ). For the decadal survey to fully evaluate the merit of a New Frontiers class Io mission, we recommend that the decadal survey perform a new mission concept study that reflects our modern view of Io and recent technological advances_- particularly with regards to radiation hardening and solar power.

Key technological advance: radiation hardening: The largest challenge for Io exploration is surviving the intense radiation environment around Jupiter. Jupiter's large, rapidly rotating magnetic field traps charged particles that can damage spacecraft electronics. Io directly contributes to this problem by injecting $\sim 1,000 \mathrm{~kg} / \mathrm{s}$ of new material (primarily $\mathrm{S}$ and $\mathrm{SO}_{2}$ gas) into the magnetosphere-which is rapidly ionized and accelerated, forming the Io plasma torus. These processes yield a deadly environment for spacecraft. Radiation forced Galileo into safe mode during half of its Io flybys (all in the late extended mission), resulting in the loss of key scientific measurements $^{21}$. A radiation-hardened spacecraft in orbit around Io would receive lethal total radiation doses ( $\sim$ megarad behind 100 millimeters of aluminum shielding) in about one month ${ }^{16}$. However, since the last decadal survey, there have been advances in our understanding of both Jupiter's radiation environment and in the methods for mitigating radiation damage, including: (1) The development of new models of the Jovian radiation environment, enabling better modeling of radiation hazards for Europa Clipper and other Jupiter missions (e.g., GIRE322); (2) The development of new methods for modeling the effect of radiation on spacecraft systems (e.g., FASTRAD, NOVICE 2017); (3) Improved strategies for spacecraft shielding (e.g., tantalum shielding ${ }^{23}$ ); (4) New measurements of the radiation environment close to Jupiter with Juno ${ }^{24}$; (5) The development of radiation-hard science electronics and instrument for BepiColombo, Europa Clipper, and JUICE, including cameras ${ }^{25-30}$, thermal imagers ${ }^{31-33}$, laser altimeters ${ }^{34}$, neutral mass spectrometers $^{35}$, and spacecraft electronics ${ }^{36-37}$. These technological advances (coupled with a thorough trade study evaluating potential trajectories, orbits, and mission architectures) could substantially mitigate the radiation risk for an Io mission compared to past studies.

Key technological advance: solar power: Solar-powered missions to 5 AU are becoming commonplace (e.g., Juno, Europa Clipper, JUICE, Lucy), and NASA has substantially invested in the development of advanced solar cell technologies ${ }^{38}$. Of particular note, NASA's Extreme Environments Solar Power (EESP) project is developing solar arrays for the low-intensity, lowtemperature, high-radiation environments of the outer solar system ${ }^{39}$. Select EESP technologies will be demonstrated on the Double Asteroid Redirect Mission (DART). These advances increase the efficacy of solar power at Io, and may enable new scientific investigations. While the requisite large solar arrays come with challenges (e.g., pointing stability), past trade studies have frequently shown that solar powered Io mission concepts outperform radioisotope power options due to the lower mass, cost, and scheduling risk ${ }^{6,10-11}$. Nonetheless, radioisotope power may enable different 
mission architectures and may be worth considering in a mission concept study.

A new, fully-costed, New Frontiers class Io mission concept study could leverage these recent technological and scientific advances, yielding a concept ripe for the next generation of Io science. A new mission concept study should perform several trade studies, including: (1) analysis of different mission architectures (e.g., multiple flyby vs. orbit) and identifying the optimal balance between cost, radiation mitigation, and achieving priority science objectives; (2) trades between science instruments; (3) the possible role of emerging technologies including new instruments (Recommendation 3) and CubeSats (Recommendation 4).

Recommendation 3: We advocate for a robust instrument and technology development pipeline for the exploration of lo. While no new technology is required for a modern Io mission, there are always new technologies that may enhance future missions and should be developed. Ref. 16 provided a prioritized list of instruments and technologies for future development, including:

- Power: Power is often a limiting factor for outer planets exploration, and more efficient solar and radioisotope power options may enable more innovative mission concepts ${ }^{38,40-41}$.

- High-dynamic range thermal imaging: Galileo observations of Io were often oversaturated due to the extreme temperature ranges on Io - from the cold surface to ultra-high temperature lavas. New detectors and electronics may enable larger dynamic ranges suitable for $\mathrm{Io}^{42}$.

- Geodetic cameras: Cameras with ultra-precise pointing would enable high-precision measurements of geophysical quantities that are otherwise inaccessible from current instruments, including topography, libration, obliquity, and Love numbers ${ }^{43}$.

- Radar sounding: Active radar sounding is a powerful geophysical tool that will soon be applied to Io's neighboring ocean worlds (REASON on Europa Clipper, RIME on JUICE). These systems are heavy, power-hungry, and susceptible to external radio noise sources like Jupiter. Passive radar may represent an appealing alternative for $\mathrm{Io}^{68}$, as these systems require less power and mass, and could use Jupiter's immense radio signals as the source, although passive systems have not been demonstrated ${ }^{44}$.

- InSAR: Interferometric Synthetic Aperture Radar has the capability to measure deformation at centimeter scales, and has been revolutionary in understanding Earth's surface processes. InSAR in the outer solar system would be transformative, but extremely challenging due to power and mission design constraints ${ }^{16}$.

- X-ray spectrometers: X-ray spectrometry is a powerful tool for measuring elemental surface abundances (e.g., XRS on MESSENGER) and could characterize the plasma torus and aurorae, although the Jovian high-radiation environment complicates the instrument design ${ }^{45-46}$.

- Seismology from orbit: Seismology is one of the most powerful methods for probing interior structure, but the challenges of landing and operating a seismometer on Io are immense. One potential solution is Laser Doppler Vibrometry, which uses a laser from a distant spacecraft to remotely measure surface vibrations via the Doppler shift of the reflected light. Seismology from orbit would be transformative, but has not been demonstrated ${ }^{47-48}$.

- Io orbiters and landers: With modern radiation hardening methods, an Io orbiter or lander might be able to survive one month ${ }^{16}$, although the required shielding increases spacecraft mass and cost. This is compounded by the large fuel requirements for getting into Io orbit or onto Io's surface, as Io is deep within Jupiter's gravity well. Impactors and penetrators may be one way to access Io's surface and interior at a lower cost than classic soft landers. Even with short lifetimes, such vehicles may enable transformative in situ science, such as seismology.

Development of these technologies may enhance future mission concepts in this decade and 
beyond. Furthermore, all of these technologies have applications beyond Io.

Recommendation 4: We advocate the use of CubeSats for high-risk, high-reward science at lo. The miniaturization of spacecraft has revolutionized the exploration of space ${ }^{49}$. These small satellites (henceforth, "CubeSats," regardless of size or form) are enabled by significant technology maturation and cost savings due to growing commercial sector demand, resulting in the ability to build spacecraft with "off the shelf" components that are capable of addressing specific scientific objectives and/or augmenting the capabilities of larger missions. Swarms and constellations of CubeSats offer a unique capability to perform distributed science observations. NASA has flown two deep space CubeSats (MarCO 1 and 2, which provided telemetry for the landing of InSight at Mars), and many others are in development.

CubeSats have the potential to address high-level science questions at Io. In particular, short-lived sacrificial CubeSats could be used to gather unique high-risk/reward datasets that may not be possible/wise/prudent for a larger, dedicated Io mission. Example concepts include probes that sample the chemistry in denser plumes, swarms that characterize the atmosphere at different times and locations, or swarms that explore Io's gravity and magnetic fields at higher spatial and temporal resolution. The use of companion CubeSats on planetary missions is still new territory, but shows significant promise. As a point of comparison, the two MarCO CubeSats onboard the InSight mission cost approximately $\$ 18.5 \mathrm{M}$, and carried onboard attitude control systems, X-band transponders, and basic cameras. We note that $\mathrm{MarCO}$ (and the other planetary CubeSats) required a ride-share, and we assume that CubeSats at Io would require a larger mothership for getting them to Io and facilitating operations (e.g., telecom). Although the spacecraft operations and radiation environment around Io are more challenging than Mars, the MarCo examples suggest that CubeSats can be credibly explored.

Recommendation 5: We advocate for continued telescopic observations of lo from ground and space-based assets, and the development of next-generation planetary astronomy telescopes capable of addressing priority lo science questions. The next generation of Earth- and space-based telescopes will enable improved sensitivity, spatial resolution, and spectral coverage of Io. These facilities and instruments may be capable of addressing some Priority Science Questions, and would complement a dedicated Io mission. Here we outline forthcoming capabilities (and strategic gaps) in planetary astronomy relevant to Io.

The James Webb Space Telescope (JWST) will have unprecedented sensitivity at wavelengths inaccessible from the ground (1-50 microns), enabling investigations of Io's atmosphere and surface frosts. However, JWST will be unable to observe Io at wavelengths longer than about 10 microns because every instrument will over-saturate due to Io's volcanoes and scattered light from Jupiter. This will inhibit JWST's ability to perform a number of key investigations, like measuring Io's heat flow.

The next generation of large ground-based telescopes (Giant Magellan Telescope, Thirty Meter Telescope, European Extremely Large Telescope) will have improved sensitivity and spatial resolution (by a factor of $\sim 3$ ) than current 8-10-meter class telescopes like Keck and the Large Binocular Telescope. The higher sensitivity and resolution mean that fainter atmospheric lines and volcanoes will be detectable, and it may be possible to observe active surface changes.

Ongoing upgrades to the Atacama Large Millimeter/submillimeter Array (ALMA) interferometer are continually increasing the sensitivity and spatial resolution of the instrument, and thermal emission can now be mapped in the radio wavelengths at the same spatial scale as reflected sunlight in the optical and infrared, enabling powerful multi-wavelength datasets for 
exploring Io's surface. $A L M A$ 's sensitivities to molecular species in tenuous atmospheres is also allowing for the detection of new trace species and mapping of more abundant molecules, and characterization of isotopic abundances ${ }^{50}$.

Proposed improvements for the next-generation Very Large Array ( $n g V L A)$ would provide unprecedented views of Io in relatively uncharted territory ${ }^{51}$. $n g V L A$ would be capable of measuring thermal properties of the surface, providing constraints on conducted heat flow and thermophysical properties of lava flows and plume deposits at resolutions comparable to Keck $(<100 \mathrm{~km}) . n g V L A$ would also be capable of mapping $\mathrm{SO}, \mathrm{SO}_{2}$, and other gas species at high resolution, that when coupled with $A L M A$ observations could yield the $3 \mathrm{D}$ temperature structure of Io's atmosphere. We endorse the development of $n g V L A$.

Planetary radar-where ground-based radio observatories transmit signals and observe their reflections - has enabled key discoveries across the solar system ${ }^{52}$. If these facilities are maintained and expanded, they could enable a variety of unique measurements, including characterizing Io's spin state, which has important implications for Io's interior structure ${ }^{53}$. We advocate for continued investment in planetary radar facilities.

The International Ultraviolet Explorer, Hubble Space Telescope, and Extreme Ultraviolet Explorer have shown that ultraviolet imaging is a powerful tool for examining plasma-satellite and plasma-Jupiter interactions. The JAXA Hisaki satellite has provided unprecedented UV monitoring of the Jovian system, although its small size precludes resolving Io. With the forthcoming retirement of Hubble, it is critically important to develop next generation telescopic assets for observing in the ultraviolet. We advocate for a large-aperture, space-based UV telescope with diffraction-limited capabilities to study Io and other planetary targets. Such a telescope could resolve Io and detect active plumes spectroscopically, observe fresh plume deposits through reflectance, resolve the spatial and temporal variability of Io's aurorae (currently on the edge of detectability with Hubble), and perhaps detect new chemical species.

Io is notoriously dynamic over a variety of timescales - from seconds to years. To understand this dynamism requires long-term, high-cadence, synoptic monitoring. Ground-based Io observation campaigns in the last decade have transformed our understanding of Io's volcanism and activity ${ }^{54-57}$, and are a critical component of exploring Io. We advocate for the continued monitoring of Io, and the development of dedicated planetary science Earth- and space-based telescopes. Even then, many questions will be difficult to resolve without a dedicated mission that can observe Io at a range of temporal and spatial scales.

Recommendation 6: We recommend a robust research and analysis program, and specifically advocate for importance of multi-disciplinary proposals that incorporate laboratory experiments and field studies relevant to lo. Research and Analysis (R\&A) are critical components of NASA's planetary science activities, and work performed under R\&A grants have transformed our understanding of Io. We advocate for a robust, well-rounded, and well-funded planetary science R\&A program.

One of the most significant gaps in R\&A funding is laboratory and field studies. Laboratory studies investigating tidal heating within planetary bodies are particularly important for understanding Io and other tidally heated worlds, and there is critical need for experiments of heating, melting, and deformation of analogue materials at temperatures, pressures, and timescales relevant to Io, ocean worlds, and other tidally-deformed worlds ${ }^{16}$. We advocate for experimental proposals and multi-disciplinary proposals including experimental and field components. Example studies could include:

- Laboratory studies of surface and atmospheric chemistry, and sulfur isotopic systems in 
particular, for determining Io's chemical evolution.

- Laboratory studies of the electrical properties of Io analogue materials (e.g., S-rich melts) as a function of melt fraction and connectivity are critical for comparison with spacecraft-measured magnetic field measurements. Electrical studies in the laboratory have proved to be a relevant tool to probe the interior of celestial bodies and the presence and distribution of molten material at depth (e.g., in the Moon and Mercury ${ }^{58-60}$ ) and would be important for interpreting Io's induced magnetic field ${ }^{61}$.

- Laboratory studies of the rheological properties of high melt fraction samples, including viscosity and anelasticity measurements under high pressure/temperature conditions. These studies would provide valuable constraints on convection and tidal heating, respectively.

- Field studies of terrestrial analogs, including active volcanic eruptions at different temporal and spatial scales ${ }^{62}$.

Recommendation 7: We recommend that the decadal survey consider the critical role of team dynamics, equity, diversity, inclusion, and accessibility in planetary science. Io exploration requires drawing on perspectives spanning the gamut of planetary science, geoscience, astronomy, technology, engineering, and beyond. Studies of scientific teams have repeatedly demonstrated the importance of an integrated approach, where team members with diverse expertise develop synergies between their specialties and resources that result in an end product that adds up to more than the sum of its parts ${ }^{63}$. We believe answering Io's Priority Science Questions requires rigorous interdisciplinarity, not only because of the scope of the questions, but because sociological studies have demonstrated that groups that foster strong connections across sub-units are more innovative ${ }^{64-66}$ with higher impact outcomes that endure ${ }^{66-67}$.

Finally, for the good of the field and for the human aspect of our endeavors, it is critical that the planetary science community fosters a diverse, equitable, inclusive, and accessible environment. We strongly encourage the decadal survey to consider the state of the profession and the issues of equity, diversity, inclusion, and accessibility - not as separable issues, but as critical steps on the pathway to understanding Io and the entire solar system. Background information on the current lack of diversity in our community and specific, actionable, and practical recommendations can be found in ref. 69-75.

\footnotetext{
${ }^{1}$ Esper et al. 2003 (10.1016/S0094-5765(02)00163-7); ${ }^{2}$ Borer et al. 2009 (2009LPI....40.1062B); ${ }^{3}$ McEwen et al. 2009 (2009LPI...40.1876M); ${ }^{4}$ Williams et al. 2009a (https://solarsystem.nasa.gov/studies/11/future-io-exploration-for-2013-2022-and-beyond-part-1-justification-and-science-objectives/); ${ }^{5}$ Williams et al. 2009b (https://solarsystem.nasa.gov/studies/12/future-io-exploration-for-2013-2022-and-beyond-part-2-recommendations-for-missions/); ${ }^{6} \mathrm{Turtle} \quad$ et al. 2010 (https://solarsystem.nasa.gov/studies/214/io-observer/); ${ }^{7}$ McEwen et al. 2010 (2010LPI...41.1433M); ${ }^{8}$ Becerra et al. 2014 (2014DPS...4621411B); ${ }^{9} \mathrm{Hays}$ et al. 2014 (2014AGUFM.P43B3977H); ${ }^{10}$ McEwen et al. 2014 (10.1016/i.actaastro.2012.05.028); ${ }^{11}$ Marcucci et al. 2015 (2015LPI...46.2624M); ${ }^{12 M c E w e n ~ e t ~ a . ~ 2015 ~(2015 L P I . . .46 .1627 M) ; ~}$ ${ }^{13}$ Keane et al. 2017 (2017LPICo1989.8161K); ${ }^{14}$ Suer et al. 2017 (10.1016/i.asr.2017.05.019); ${ }^{15}$ McEwen et al. 2019 (2019LPI...50.1316M); ${ }^{16}$ de Kleer et al. 2019c (https://www.kiss.caltech.edu/final reports/tidal heating final report.pdf); ${ }^{17}$ Turtle et al. 2020 (2020LPI...51.2288T); ${ }^{18}$ NRC 2011 (10.17226/13117); ${ }^{19} \mathrm{NRC} 2017$ (10.17226/24843); ${ }^{20}$ NRC 2018 (10.17226/25186); ${ }^{21}$ Lopes \& Spencer 2007 (10.1007/978-3-540-48841-5); ${ }^{22}$ Garrett et al. 2017 (10.1109/TNS.2017.2755618); ${ }^{23}$ Vuolo et al. 2016 (Topical Day for RADECS Conference, B., Vol. 16); ${ }^{24}$ Becker 2017 (10.1002/2017GL073091); ${ }^{25}$ Centurelli et al. 2018 (10.1117/12.2313596); ${ }^{26}$ Janesick et al. 2014 (10.1117/12.2063524); ${ }^{27}$ McEwen et al. 2012 (2012LPICo1683.1041M); ${ }^{28}$ McEwen et al. 2014 (10.1016/i.actaastro.2012.05.028); ${ }^{29}$ Michaelis et al. 2017 (10.1117/12.2304114); ${ }^{30}$ Turtle et al. 2016 (2016LPIC01980.4091T); ${ }^{31}$ Dartois et al. 2017 (10.1117/12.2279413); ${ }^{32}$ Gaalema et al. 2010 (10.1117/12.859726); ${ }^{33}$ Hiesinger et al. 2010 (10.1016/i.pss.2008.09.019); ${ }^{34}$ Althaus et al. 2016 (2016LPICo1980.4015A); ${ }^{35} T u l e j$ et al. 2016 (10.1016/i.nimb.2016.06.008); ${ }^{36} \mathrm{Furano}$ et al. 2013 (10.1088/17480221/8/02/C02007); ${ }^{37}$ Cressler \& Mantooth 2017 (10.1201/b13001); ${ }^{38}$ NASA 2017 (https://solarsystem.nasa.gov/resources/548/solar-power-technologies-for-future-planetaryscience-missions/); ${ }^{39} \mathrm{EESP}$ (https://gameon.nasa.gov/extreme-environments-solar-power/). ${ }^{40}$ Sherwood et al. 2018 (10.1016/i.actaastro.2017.11.047); ${ }^{41} \mathrm{NRC} 2012$ (10.17226/13354); ${ }^{42}$ Davies et al. 2016 (10.1016/i.icarus.2016.06.003); ${ }^{43}$ Park et al. 2016 (2016LPICo1980.4018P); ${ }^{44}$ Steinbrügge et al. 2018 (10.1016/i.epsl.2017.11.028); ${ }^{45}$ Bhardwaj et al. 2007 (10.1016/i.pss.2006.11.009); ${ }^{46}$ Elsner et al. 2005 (10.1016/i.icarus.2005.06.006); ${ }^{47}$ Sava \& Asphaug 2019a (2019LPI....50.1709S); ${ }^{48}$ Sava \& Asphaug 2019b (10.1016/i.asr.2019.04.017); ${ }^{49}$ NRC 2016 (10.17226/23503); ${ }^{50}$ Moullet et al. 2013 (10.1088/0004-637X/776/1/32;); ${ }^{51}$ de Kleer et al. 2020 (white paper: ngVLA); ${ }^{52}$ RiveraValentín et al. 2020a (white paper: planetary radar); ${ }^{53}$ Margot et al. 2013 (2012AGUFM.P54A..02M); ${ }^{54}$ Cantrall et al. 2018 (10.1016/i.icarus.2018.04.007); ${ }^{55} \mathrm{de} \mathrm{Pater} \mathrm{et} \mathrm{al.} \mathrm{2017}$ (/10.1016/j.icarus.2017.03.016); ${ }^{56}$ de Kleer \& de Pater 2016a (10.1016/i.icarus.2016.06.018); ${ }^{57}$ de Kleer \& de Pater 2016b (10.1016/j.icarus.2016.06.019); ${ }^{58}$ Pommier et al. 2015a (10.1038/nature14502); ${ }^{59}$ Pommier et al. 2015a (10.1016/i.epsl.2015.05.052); ${ }^{60}$ Pommier et al. 2019 (10.1016/i.epsl.2019.04.022); ${ }^{61} \mathrm{Khurana}$ et al. 2011 (10.1126/science.1201425); ${ }^{62}$ Radebaugh et al. 2016 (10.1016/i.jvolgeores.2016.03.019); ${ }^{63}$ Balakrishan et al. 2011 (10.1145/1958824); ${ }^{64}$ Burt 2004 (10.1086/421787); ${ }^{65}$ Powell et al. 1996 (10.2307/2393988); ${ }^{66}$ de Vaan et al. 2015 (10.1086/681213); ${ }^{67}$ Curral et al. 2001 (10.1080/13594320143000627); ${ }^{68}$ Steinbrügge et al. 2020 (white paper: PRIME); ${ }^{69}$ Rivera-Valentín et al. 2020b (white paper: who is missing in planetary science, part 1); ${ }^{70}$ Rathbun et al. 2020 (white paper: who is missing in planetary science, part 2); ${ }^{71}$ Strauss et al. 2020 (white paper: non-binary inclusion in planetary science); ${ }^{72}$ Milazzo et al. 2020 (white paper: DEIA 101); ${ }^{73}$ Piatek et al. 2020 (white paper: accessibility within planetary science); ${ }^{74}$ Rivera-Valentín et al. 2020c (white paper: LGBTQ+ in planetary science); ${ }^{75}$ Richey et al 2020 (white paper: lessons from the Astro2020 decadal survey).
}

Additional resources available at www.jamestuttlekeane.com/io. 